zeigt, daß auf diesem Wege eine weitere Möglichkeit zur Deutung des Zustandes flüssiger Verbindungen gegeben ist.

Die ermittelten Atomverteilungskurven der flüssigen Legierung I und der flüssigen Verbindung zeigen keine besonderen Merkmale und gleichen eher der einer reinen Metallschmelze, wie z. B. der Kurve des flüssigen Germaniums (Abb.18). Die $W$-Kurve des flüssigen Eutektikums fällt dagegen durch ein extrem hohes und schlankes erstes Maximum auf ( $W=3,7$ ), dem eine sehr scharfe Senke bei $\boldsymbol{r}=3,5 \AA$ folgt. Allen drei Kurven gemeinsam ist ein im Abstand von 3,85 bis $3,88 \AA$ sich abhebendes schwaches Maximum.

Während die Deutung der Interferenzbilder gewisse Aussagen ermöglicht, ergibt sich aus den entsprechenden Atomverteilungskurven lediglich ein mittlerer kleinster Atomabstand (Tab.4). Die-

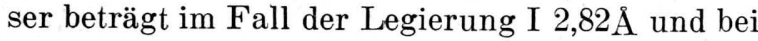
der Legierung II und der Verbindung AuSn 2,85 A. Diese Atomabstände sind somit kleiner als der bei einer Koordinationszahl 11 im reinen Gold gefun- dene. Eine exakte Zuordnung ist jedoch nicht möglich. Z. B. ist in der festen Verbindung AuSn der Abstand von Gold- zu Gold-Atom bei einer. Zweierkoordination 2,75 A und der Abstand von Gold- zu Zinn-Atom bei einer Sechserkoordination 2,84. Inwieweit noch der Abstand der ZinnAtome untereinander, der etwa 3,1 bis $3,2 \AA$ beträgt, an der Bildung der ersten Koordination beteiligt ist, läßt sich nicht beurteilen.

Wegen der nahezu gleichen Atomabstände Gold-Gold, Gold-Zinn und Zinn-Zinn ist eine Auswertung der Fläche unter dem ersten Maximum der Atomverteilungskurven der flüssigen Legierungen nicht möglich, so daß über die Art und die Zahl der nächsten Nachbarn keine Aussage gemacht werden kann. Binäre Systeme mit großem Unterschied der Atomradien dürften hierfür günstiger sein.

Hrn. Prof. Dr. R. G l o ck e r möchte ich an dieser Stelle herzlich danken für das große Interesse an dieser Arbeit und deren stete Förderung, die die Durchführung derselben in den Kriegsjahren ermöglichte.

\title{
Zur Fernausbreitung der Kurzwellen
}

\author{
Von Ludwig Hamberger und Karl Rawer \\ Mit einem Nachwort von H. A. Hef \\ (7. Naturforschg. 2 a, 521-527 [1947]; aus Neuershausen bei Freiburg i. Br. eingegangen am 15. April 1947)
}

Die erstaunlich konstante Umlaufzeit der Kurzwellen um den Erdball läßt sich, entgegen der Ansicht von $\mathrm{H}$. A. He B, auch mit der Vorstellung einer Zickzack-Reflexion zwischen Ionosphäre und Erde erklären. Die in dem Bericht von $\mathrm{H}$ e $\&$ mitgeteilten Umlaufzeiten machen es wahrscheinlich, daß die Zeichen 12- bis 17-mal zwischen Erde und Ionosphäre hin und her reflektiert werden.

$\mathrm{I}$ Tn dieser Zeitschrift hat kürzlich H. A. $\mathrm{He} ß^{1}$ Iüber Untersuchungen an Kurzwellen-EchoSignalen berichtet. Er gibt darin im wesentlichen den Inhalt eines ZWB-Berichtes ${ }^{2}$ wieder, der während des Krieges im Institut von Prof. O. v. $\mathrm{Sch}$ mid t entstanden war. Schon seinerzeit hatte dazu die Zentralstelle für Funkberatung (Leitung W. Di e ming e r) in einem Bericht der Verfasser dieses Aufsatzes ${ }^{3}$ Stellung genommen.

1 H. A. He ß, Z. Naturforschg. 1, 499 [1946].

${ }^{2}$ H. A. Heß, O. v. S ch mid t u. G. Schultze, Indirekte Zeichen und Erdumläufe, Dtsch. Luftfahrtforschg. FB Nr. 1898 [1943]. Im folgenden kurz „Originalbericht" genannt.

${ }^{3}$ L. H a m b e r g e r u. K. R a w er, Die Fernausbreitung der Kurzwellen über Zickzackwege, Dtsch. Luftfahrtforschg. U'M Nr. 789 [1944].
H. A. H e $B$ faßt seine Beobachtungsergebnisse in vier Feststellungen zusammen, die seiner Ansicht nach nicht mit der Vorstellung einer Ausbreitung der Kurzwellen auf Zickzack-Wegen zwischen Erde und Ionosphäre vereinbar sind:

1. Die empfangenen Zeichen sind um so klarer, je größer der durchlaufene Weg ist. Das schließt aus, daß Mehrfachwege auftreten.

2. Das Feldstärkeverhältnis der direkten und indirekten Zeichen und der Umläufe ist für die Zeichen längeren Weges günstiger als erwartet. 3. Die Laufzeit des Kurzwellenumlaufs ist praktisch konstant und unabhängig von der Frequenz, von der Tages- und Jahreszeit sowie von der Sonnenflecken-Periode.
Dieses Werk wurde im Jahr 2013 vom Verlag Zeitschrift für Naturforschung in Zusammenarbeit mit der Max-Planck-Gesellschaft zur Förderung der Wissenschaften e.V. digitalisiert und unter folgender Lizenz veröffentlicht: Creative Commons Namensnennung-Keine Bearbeitung 3.0 Deutschland Lizenz.

Zum 01.01.2015 ist eine Anpassung der Lizenzbedingungen (Entfall der Creative Commons Lizenzbedingung „Keine Bearbeitung“) beabsichtigt, um eine Nachnutzung auch im Rahmen zukünftiger wissenschaftlicher Nutzungsformen zu ermöglichen.
This work has been digitalized and published in 2013 by Verlag Zeitschrift für Naturforschung in cooperation with the Max Planck Society for the Advancement of Science under a Creative Commons Attribution-NoDerivs 3.0 Germany License.

On 01.01.2015 it is planned to change the License Conditions (the removal of the Creative Commons License condition "no derivative works"). This is to allow reuse in the area of future scientific usage. 
4. Von Telefunken angestellte Messungen ${ }^{4}$ haben Einfallswinkel im Fernverkehr von etwa $20^{\circ}$ ergeben. Heß nimmt an, daß auch die Umlaufzeichen derart steil einfallen. Die Vorstellung eines Zickzack-Weges kann in diesem Fall die gemessenen Laufzeiten nicht erklären.

Im folgenden werden wir nicht auf die v.Sch midtsche Theorie der Kopfwellenausbreitung eingehen. Es wird lediglich versucht werden, nachzuweisen, daß alle Beobachtungsergebnisse, insbeson-

- dere die von Heß unter 1.-3. genannten, sich zwanglos durch Zickzack-Reflexionen erklären lassen. Die von $\mathrm{He} ß$ unter 4. angezogenen Ergebnisse schließlich treffen im vorliegenden Falle nicht zu. Für alle Rechnungen setzen wir scharfe Spiegelung an der Ionosphäre voraus, was eine recht gute Näherung ist ${ }^{5}$.

Zu 1. Eine Verbindung durch Spiegelung an der Ionosphäre kommt auf einem bestimmten Zickzack-Weg nur dann zustande, wenn folgende drei Bedingungen erfüllt sind:

a) An der Reflexionsstelle muß die Elektronendichte zur Spiegelung ausreichen; die „Grenzfrequenz" der Spiegelung hängt vom Einfallswinkel ab.

b) Die Dämpfung, welche die Welle beim Durchlaufen der niederen Ionosphärenschichten erleidet, muß so gering sein, daß am Empfangsort noch eine ausreichende Feldstärke ankommt ${ }^{6}$.

c) Erfolgt die Spiegelung (wie gewöhnlich bei Weitstrecken) an der $F$-Schicht, so muß die „Abdeckung“ vermieden werden, d. h. die Trägerdichte der $E$-Schicht darf noch nicht zur Reflexion ausreichen ${ }^{6}$.

Diese Auswahlbedingungen grenzen den brauchbaren Frequenz- und Winkelbereich ein. Die Bedingungen der Abdeckung und Dämpfung fordern genügend hohe, die der Spiegelung nicht zu hohe Frequenz.

Erfolgt die Ausbreitung in mehreren Schritten, so muß die erste Bedingung an allen Reflexionspunkten, die dritte an allen Durchstoßstellen durch die $E$-Schicht erfüllt sein ${ }^{6}$. Die zweite Bedingung bedeutet jetzt, daß die Summe der Dämp-

${ }_{4}$ E. S chüttlöffel u. G. Vogt, VDE-Fachbericht 11, 48 [1939]; P. K o t ow s ki, E. Schüt $\mathrm{l}$ ö f e l u. G. Vo g t, Kurzwellenanlagen mit steuerbarer Richtcharakteristik und ihre Anwendung zur Messung von Einfallswinkeln, Mitt. Telefunken-Labor. [1940]. fungseinflüsse an allen Stellen, wo die $D$-Schicht durchsetzt wird, zusammen mit der Verdünnung der Energie durch die Entfernung, ein Höchstmaß nicht überschreiten darf (das durch die Leiştung der Geräte festgelegt ist). Je größer nun die Entfernung, um so verschiedener ist der $\mathrm{Zu}$ stand der Ionosphäre längs der Strecke, um so enger grenzen die Auswahlbedingungen die Möglichkeiten einer Verbindung ein. Die Erfahrung zeigt, daß reine Tag- oder reine Nachtstrecken immer leicht überbrückt werden. Auf gemischten Strecken jedoch widersprechen sich die Forderungen sehr häufig; die für die Nachtseite geforderten niedrigen Frequenzen werden auf der Tagseite zu stark gedämpft und abgedeckt; umgekehrt reicht für die hohen Tagfrequenzen die Elektronendichte der Nachtseite nicht mehr zur Reflexion aus. Ein Weg um die Erde herum wird immer Tag- und Nachtseite durchsetzen, es sei denn, er verläuft im Dämmerungsgürtel; nur dort hat man geringe Dämpfung und gleichzeitig ausreichende Grenzfrequenz. Tatsächlich werden Erdumläufe meist im Dämmerungsgürtel beobachtet.

Die Auswahlbedingungen sind bei gegebener Strecke auch der Schrittzahl nach verschieden. Wege in vielen Schritten verlaufen steil; die (dritte) Bedingung der Abdeckung ist hier harmlos, aber die Dämpfung ist groß, weil die $D$-Schicht vielmals durchsetzt wird; außerdem ist die Reflexionsbedingung für steile Strahlen ungünstig. Umgekehrt verlaufen Wege kleiner Schrittzahl flach; hier sind die erste und zweite Bedingung leichter zu erfüllen als die dritte der Abdeckung. So werden nur bestimmte Wege jeweils möglich sein; welche, hängt.von Frequenz und Ionosphärenzustand ab. Je größer nun die Entfernung wird, um so seltener werden die Bedingungen für verschiedene Wege gleichzeitig erfüllt sein. Bei Erdumläufen sind die Bedingungen meist überhaupt nicht zu erfüllen; wenn aber einmal, so nur für einen bestimmten Weg. Deshalb sind hier Mehrfachzeichen gar nicht zu erwarten, auch von der Vorstellung eines ZickzackWeges aus.

$\mathrm{Zu}$ 2. Die von $\mathrm{He} B$ angegebenen Amplituden der indirekten und Umlaufzeichen sind nicht überraschend. Im mitgeteilten Beispiel (Südame-

5 N. S m i t h, J. Res. nat. Bur. Standards 20 [1938], May.

${ }_{6}^{6}$ K. R a w e r, Der Einfluß der Dämpfung auf die Kurzwellen-Ausbreitung, Dtsch. Luftfahrtforschg. FB Nr. 1872 [1943]. 
rika) war die Amplitude des Umlaufs $20 \%$ des direkten Zeichens, das Wegeverhältnis $5: 1$, also genau reziprok. Daraus würde sich eine Feldstärkeabnahme mit $1 / r$ ergeben. Nach den von $\mathrm{R}$ a w e ${ }^{6}$ ausgearbeiteten Vorstellungen hat man eine Verringerung der Feldstärke durch Verdünnung einerseits und Dämpfung (in tiefen Schichten) andererseits zu erwarten; die Reflexion selbst ist praktisch verlustlos. Bei ebener Ionosphäre und Erde hätte man durch Verdünnung einen Feldstärkeabfall nach $1 / r \quad \mathrm{zu}$ erwarten. Dieser wird durch die Dämpfung verschärft, die im Däm-
Es ergibt sich auch hier kein grundsätzlicher Widerspruch zwischen der Beobachtung und der Zickzack-Theorie.

$\mathrm{Zu}$ 3. Die „Konstanz der Umlaufzeit“ ist tatsächlich bemerkenswert. Von Schmidt und seine Mitarb. haben die Versuche zweifellos sehr exakt, einwandfrei und reproduzierbar durchgeführt. Der Originalbericht gibt die Meßwerte in Tabellen, aus denen die statistische Verteilung erhellt wird. Man kann indirekte Zeichen und Umläufe miteinander vergleichen, wenn man aus den Laufzeiten die scheinbare Geschwindigkeit bzw. den

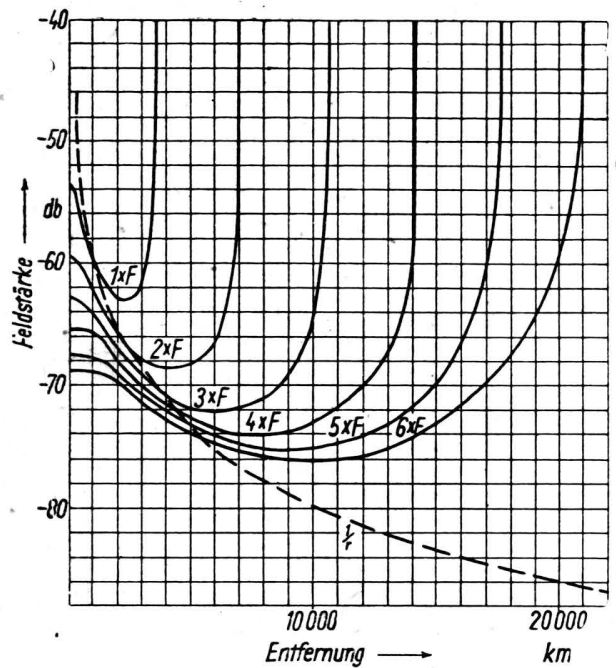

Abb. 1. Feldstärke der Raumwelle ohne Dämpfungseinfluß. (Ausgezogene Kurven mit Fokussierung nach $\mathrm{R}$ a w e r, berechnet für Schichthöhe $250 \mathrm{~km}$. Abfall nach $1 / r$ zum Vergleich.)

merungsgürtel aber nicht sehr stark ist. Andererseits aber hat R a w e r festgestellt, daß die Kugelgestalt von Erde und Ionosphäre für flache $\mathrm{Ab}$ strahlung zu einer Fokussierung führt. Sie bewirkt zumindest eine wesentlich langsamere $\mathrm{Ab}$ nahme der Feldstärke. Erst diese Fokussierung erklärt die beobachteten Reichweiten der Kurzwellen. Abb. 1 zeigt für verschiedene Wege die Feldstärke in decibel in Abhängigkeit von der Entfernung nach $\mathrm{R}$ aw e r (Schichthöhe $250 \mathrm{~km}$ ) und nach dem $1 / r$-Gesetz. Die Fokussierung findet jeweils für streifenden Strahlengang statt. Flache Abgangswinkel sind also bevorzugt. Auch wenn, etwa wegen der Unebenheiten von Erde und Ionosphäre, nicht die volle Fokussierung erreicht wird, ist eine erhebliche Feldverbesserung sicher.

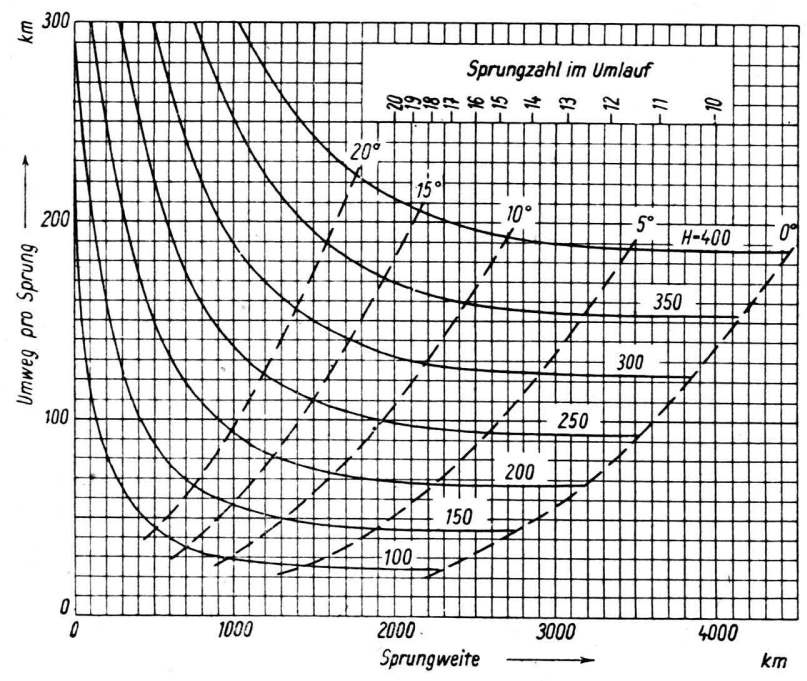

Abb.2. Umweg der Raumwelle für verschiedene Sprungweiten. (Parameter der ausgezogenen Kurven die Reflexionshöhe, der gestrichelten Kurven der Einfallswinkel.)

Unwegfaktor (Lichtgeschwindigkeit durch scheinbare Geschwindigkeit) errechnet (vergl. Abb.5 u. 6). Die indirekten Zeichen zeigen insgesamt eine „Halbwertsbreite“ der Verteilung von etwa $\pm 3 \%$, die Umläufe sogar von $\pm 2 \%$. In der Umlaufzeit entspricht das $\pm 0,4$ bzw. 0,3 msec oder Wegdifferenzen von rund $\pm 100 \mathrm{~km}$. Die Punkte größter Abweichung im Originalbericht zeigen allerdings den zehnfachen Wert. Die vorangegangene Diskussion hat jedoch ergeben, daß Umlaufsmessungen an nahen (deutschen) Stationen systematische Abweichungen ergeben müssen. Scheidet man diese aus ${ }^{7}$, so ergibt sich ungefähr

7 H. A. H e B, O. v. Sch midt u. G. Sch u l t z e, Antwort auf die Stellungnahme der Zentralstelle f. Funkberatung, Dtsch. Luftfahrtforschg. UM Nr. 789 [1944]. 
eine Gau ßsche Verteilungskurve (Abb.6), zumindest für die Umläufe.

Diese Konstanz folgt jedoch auch aus der Zickzack-Vorstellung, allerdings nur, wenn ziemlich flache Einfallswinkel vorliegen. Das ergibt die einfache geometrische Berechnung der ZickzackWege. Abb. 2 zeigt für verschiedene Schichthöhen jeweils den Umweg (Zickzack-Weg minus Entfernung) in Abhängigkeit von der Sprungweite, dazu den jeweiligen Einfallswinkel. Der Umweg ist für große Sprungweite (flachen Einfall) nicht groß (um $100 \mathrm{~km}$ pro Sprung), vor allem aber hängt er dort nicht mehr von der Sprungweite ab.

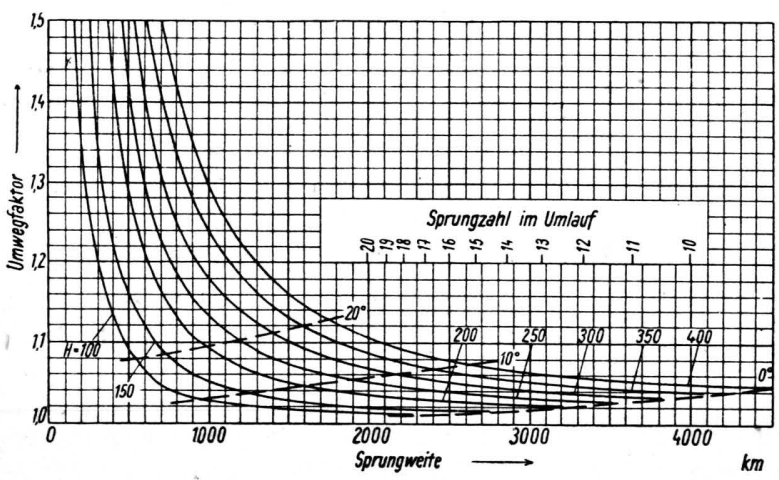

Abb. 3. Umwegfaktor für verschiedene Sprungweiten. (Parameter der ausgezogenen Kurven die Reflexionshöhe, der gestrichelten Kurven der Einfallswinkel.)

Für Einfallswinkel unter $10^{\circ}$ betragen die Unterschiede im Umweg nur noch wenige $\mathrm{km}$ ! Es bleibt lediglich eine Abhängigkeit von der Schichthöhe. Genauer zeigt das noch Abb.3, wo der Umwegfaktor (Zickzack-Weg durch Entfernung) gebildet ist. Er ist für flache Wege erstaunlich wenig von 1 verschieden. Rechnet man mit Spiegelung an der $F$-Schicht und einer wirksamen Höhe zwischen 200 und $300 \mathrm{~km}$, so liegt der Umwegfaktor zwischen 1,02 und 1,06, sofern der Einfallswinkel weniger als $10^{\circ}$ beträgt. (Sollte dagegen der Winkel zwischen $10^{\circ}$ und $20^{\circ}$ liegen, so ist der Bereich größer, von 1,045 bis 1,10 .) Selbst bei dieser sehr groben Abschätzung der Reflexionshöhe ergibt sich also im Umwegfaktor nur ein recht geringer Streubereich.

Tatsächlich darf man bei Umläufen wesentlich kleinere Höhenschwankungen ansetzen. In Wirklichkeit ist die wirksame Höhe nicht an allen Reflexionsstellen dieselbe; es liegt ja je die Hälfte im Tagbereich und im Nachtbereich, je die Hälfte auf der Nord- und auf der Südhalbkugel. In die Rechnung geht im wesentlichen die Summe der Reflexionshöhen ein; sie ist sicher sehr viel weniger veränderlich als der einzelne Wert. Auch zeitliche Schwankungen werden sich weitgehend ausmitteln.

Für Umläufe ist in Abb. 4 der Zusammenhang zwischen Einfallswinkel und Umwegfaktor wiedergegeben; Parameter der Kurven ist die Schrittzahl für den Erdumlauf. Die Kurven liegen dichtgedrängt, Übergang von einer Schrittzahl zur nächsten (bei fester Höhe) hat nur eine geringfügige Veränderung im Umwegfaktor zur Folge.

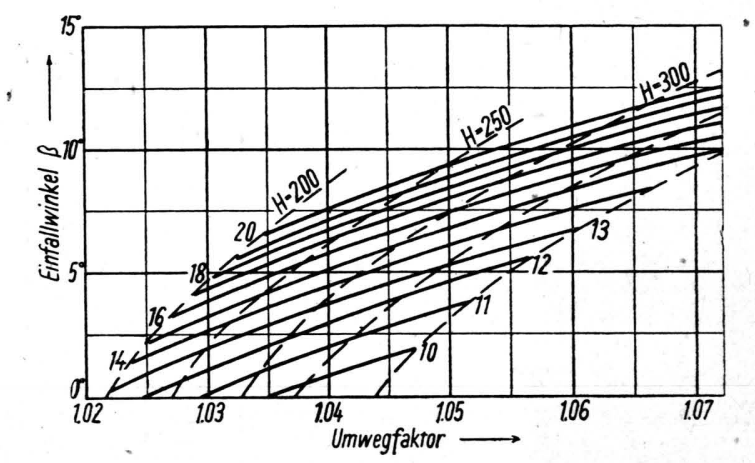

Abb. 4. Einfallswinkel und Umwegfaktor für Umlaufzeichen. (Parameter die Sprungzahl. Gestrichelt: Kurven gleicher Reflexionshöhe.)

Wollte man etwa in dem von $\mathrm{He} \cap$ wiedergegebenen Meßbeispiel (in Abb. 5 unten) die Variation durch Schrittzahlveränderung erklären (auch bei indirekten Zeichen wird ja die ganze Erde einmal umspannt), so ergäben sich etwa 12 bis 15 Schritte, also eine Veränderung um drei, innerhalb einer ganz kurzen Zeit (etwa 1 min). Man ersieht daraus, wie wenig bei flachen Wegen die Schrittzahl eingeht. Höhe und Einfallswinkel sind wichtiger.

In Abb. 5 sind nach dem Originalbericht die Ergebnisse für eine Reihe von fernen Stationen zusammengestellt. Abb. 6 zeigt nochmals die zusammenfassende Verteilungskurve für diese indirekten Zeichen, dazu die der Umläufe $\left(\right.$ nach $\left.^{7}\right)$; darunter ist der Zusammenhang zwischen Umwegfaktor, Schichthöhe und Einfallswinkel für die verschiedenen Schrittzahlen wiedergegeben. Man kann also hier unmittelbar vergleichen. Der häufigste Wert des Umwegfaktors ist 1,033. Er ist mit Schrittzahlen zwischen 11 und 18 und entspre- 
chenden Reflexionshöhen zwischen 300 und $210 \mathrm{~km}$ realisierbar; Änderung der Schrittzahl um 1·kann durch eine Höhenänderung um nur $10 \mathrm{~km}$ kompensiert werden. Die beobachtete Streuung der Umlaufzeiten ist - so geringfügig sie tatsächlich ist - immer noch groß genug, um recht erhebliche Änderungen von Höhe wie Schrittzahl zuzulassen; erstere könnte bei konstanter Höhe von 12 bis 17 variieren, letztere bei fester Schrittzahl (14) von 200 bis $280 \mathrm{~km}$. Wollte man umgekehrt versuchen, aus den Beobachtungen beides zu bestimmen; so wären nur recht vage Schlüsse möglich. Dagegen läßt sich der Einfallswinkel in ziemlich engen Grenzen festlegen; er muß zwischen $0^{\circ}$ und $7^{\circ}$ liegen. Die erwähnte Fokussierung für streifende Strahlen ${ }^{6}$ gibt eine Möglichkeit, diese Bevorzugung der flachen Wege zu erklären.

Die übliçhen Senkrechtlotungen zeigen schon, daß zwischen Laufzeit und Frequenz durchaus kein monotoner Zusammenhang bestehen muß. Neben der Höhenzunahme bei Annäherung an die Grenzfrequenz steht die Verzögerung in der $E$ Schicht bei Annäherung an den Abdeckungsfall. Da ein Umlauf insgesamt sehr verschiedene Ionosphärenzustände durchläuft, werden sich die Einfiüsse ebenfalls weitgehend ausmitteln, so daß kein systematischer Gang mit der Frequenz zu erwarten ist.

Ein Einfluß der Tages- und Jahreszeit besteht für die Häufigkeit der Umläufe, nicht aber für ihre Lanfzeit. Auch das folgt daraus, daß jedes Umlaufzeichen verschiedene Ionosphärenzustände durchläuft und der Umwegfaktor nur geringfügig von Höhenänderungen abhängig ist. Tatsächlich durchläuft ein Umlaufzeichen alle Tageszeiten. Die eine Hälfte seines Weges liegt auf der Nordhalbkugel, die andere Hälfte auf der Südhalbkugel; der Jahreszeit nach stehen diese in Opposition, was wiederum eine Ausmittelung bedeutet.

Die v.Schmidtschen Versuche sind durchweg im Minimum des Sonnenfleckenzyklus durchgeführt worden. Der Frequenzbereich, der Echos zeigt, wird sich zwar entsprechend der Sonnenfleckenperiode verschieben, die Reflexionshöhe wird sich aber nicht verändern, weil nur die Trägerdichte mit der Periode geht. Nachdem erfahrungsgemäß der Anstieg der Grenzfrequenzen der $\mathrm{F}_{2}$-Schicht erheblich stärker ist als die Zunahme von Dämpfung und Abdeckung, kann man allgemein eine Zunahme der Echotätigkeit im jetzigen
Maximum des Zyklus erwarten; damit vielleicht auch eine etwas größere Streuung in den Umlaufzeiten, da ja die Auswahlbedingungen nicht mehr so kritisch sind wie im Minimum.

Hier wäre auch noch auf die Umlaufmessungen. an nahen Stationen einzugehen, die in der er-

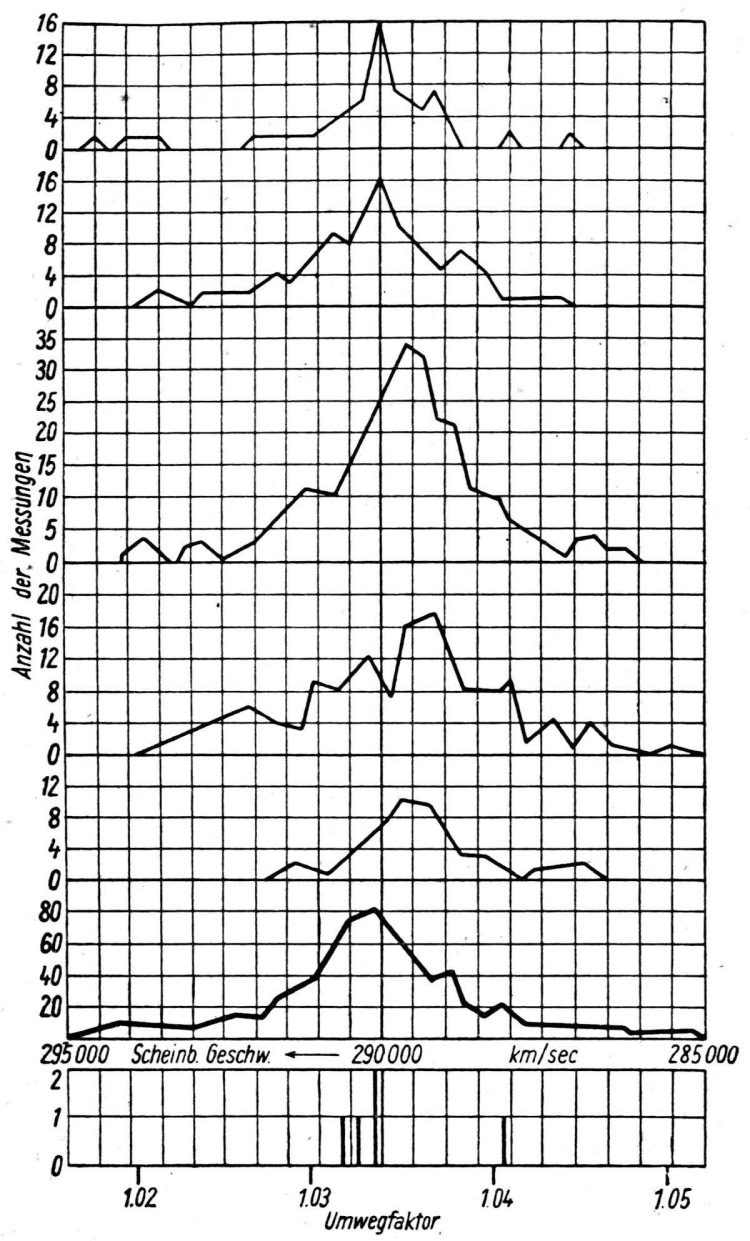

Abb. 5. Statistik der scheinbaren Geschwindigkeiten indirekter Zeichen (nach He \& u. Schultze).

wähnten Diskussion eine besondere Rolle spielten. Es ergab sich dabei allgemein eine erheblich kürzere Zeit für den ersten Umlauf und außerdem eine stärkere Streuung; die mehrfachen Umläufe hatten wieder die gewohnten Zeitdifferenzen. Auch diese Erscheinung erklärt sich zwanglos aus der Zickzack-Vorstellung. Die erste Zeit ist die Differenz zwischen einem Fernzeichen und einem Nahzeichen; letzteres hat einen wesentlich größeren Umwegfaktor. Für die Differenz ergibt sich darum ein kleinerer Umwegfaktor, als er bei 
her noch nicht gemacht worden; derartige Messungen könnten eine Entscheidung ergeben.

Eine vollständige Klärung der AusbreitungsErscheinungen erfordert Versuche mit Impulsen auf große Entfernung. Die bisherigen langjährigen deutschen Beobachtungen ${ }^{9}$ gingen über mittlere Entfernungen von etwa $500 \mathrm{~km}$ bis $1000 \mathrm{~km}$. Ihre eingehende Auswertung ergab keine Zeichen, die sich nicht der Zickzack-Vorstellung

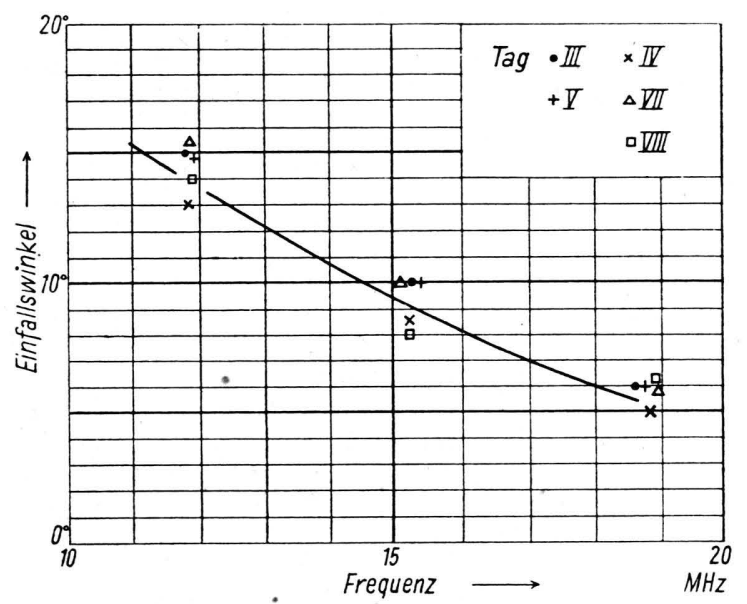

Abb. 7. Einfallswinkel im Transatlantikverkehr für drei Frequenzen nach Messungen von N e y e r. (Ausgezählte Mittelwerte, Messungen von 1944.)

eingefügt hätten. Auch die von $\mathrm{He} ß$ berichteten Ergebnisse lassen sich damit erklären. Es scheint uns daher kein experimenteller Grund zur Annahme einer zusätzlichen Kopf welle zu bestehen ${ }^{\mathbf{1 0}}$.

\section{Nachwort}

von $\mathrm{Hanns}$ Albrecht Heß

Während der Abfassung der Abhandlung ,U n tersuchungen an Kurzwellen-Echosignale $n^{" 11}$ waren dem Verf. die wesentlichen

9 W. Crone, M. Kr ̈̈ger, G. Goubau u. J. $\mathrm{Z}$ e n n e ck, Hochfrequenztechn. u. Elektroakust. 49, 1 [1936]; R. E y f r i g, Hochfrequenztechn. u. Elektroakust. 56, 161 [1940]. W. D i em ing er u. K. R a w e r, Ergebnisse von Impulsübertragungsversuchen bei Kurzwellen, Dtsch. Luftfahrtforschg. FB 1809 [1943].
Einzelheiten des ZWB-Berichtes von $\mathrm{H}$ a $\mathrm{m}$ be r ger und Rawer ${ }^{12}$ infolge der durch den Krieg bedingten Verhältnisse noch nicht zur Kenntnis gelangt. Bei der Anwendung der Kopfwellentheorie auf die ionosphärische Kurzwellenausbreitung über große Entfernungen stützte sich O.v.Sch m id t auch auf fremde Messungen über Einfallswinkel. Die im Jahre 1939 von K o tow ski gefundenen Werte von etwa $20^{\circ}$ widersprachen der Annahme von Zickzack-Reflexionen bei Erdumläufen und schienen die Kopfwellenanschauung zu bekräftigen, da unter einem bestimmten steileren Winkel Totalreflexion angenommen werden konnte. Außer acht gelassen wurde dabei, daß diese Messungen von Kotowski während des Sonnenfleckenmaximums bei anderen ionosphärischen Verhältnissen stattfanden. Die von H. N e y e r im Jahre 1944 durchgeführten Einfallswinkel-Messungen an nordamerikanischen $\mathrm{KW}$.-Sendern zeigten aber zumeist kleinere Winkel zwischen 5 und $10^{\circ}$. Dies dürfte immerhin ein wesentliches Argument für die Zickzack-Reflexionen bedeuten, wenn auch auf Grund der Berechnungen, unter Berücksichtigung der sehr konstanten Umlaufszeit, die Winkel bei Erdumläufen zwischen $0^{\circ}$ und $5^{\circ}$ liegen sollten. Messungen der Einfallswinkel von Erdumläufen mögen hier entscheiden.

Da Oswald v. Schmidt den Krieg nicht überlebt hat und sich in der Aufdeckung der Bedeutung der Kopfwelle bei vielen Wellenausbreitungen in der Akustik und Optik unvergängliche Verdienste erworben hatte, hielt es der Verf. als ehemaliger Mitarbeiter für seine Aufgabe, seine oft sehr wertvollen Ideen darch eine Veröffentlichung weiterzuleiten, obwohl ihm gewisse Einwände bekannt waren.

Ulm (Donau), den 10. Juni 1947.

Schadstr. 24.

10 Wie uns Hr. G. S ch u l t z e brieflich mitteilt, bezweifelte O. v. Sch m idt das Auftreten von Zickzackwegen nicht. Er behauptete lediglich, es trete zusätzlich für große Entfernungen eine „Kopfwelle“ auf.

11 Z. Naturforschg. 1, 499 [1946].

12 Dtsch. Luftfahrtforschg. UM 789 [1944]. 